\title{
Secretion of Hepatic Triglycerides into Plasma of Rats Fed Retinol
}

\author{
H. C. Ahuja and U. K. Misra \\ Department of Radioisotopes and Biochemistry, Vallabhbhai Patel Chest Institute, \\ University of Delhi, Delhi-7, India
}

Received September 2, 1974

\begin{abstract}
The effect of feeding $33 \mathrm{mg}$ of retinol daily for two days on liver and plasma lipids of rats has been studied. The secretion of liver TG into plasma of retinol fed rats has been measured by the use of palmitic acid-1-14 $\mathrm{C}$ and of Triton WR 1339. Liver and plasma lipids, TG, phospholipids and PC were significantly higher in retinol fed rats as compared to control rats. The incorporation of palmitic acid-1-14C into liver TG, PC and PE was significantly higher in retinol fed rats. The labelling pattern with time of liver and plasma TG and PC shows that the secretion of liver TG and PC into plasma was impaired in retinol fed rats.
\end{abstract}

Oral or intramuscular administration of large amounts of vitamin A to experimental animals causes several characteristic abnormalities." One of the more pronounced effect of hypervitaminosis $A$ is a large increase in liver triglycerides accompanied by hypertriglyceridemia and hyperphospholipedemia. ${ }^{2 \sim 5)}$ Since no studies have been done to explain the mechanism of these effects the present study was undertaken to study the synthesis of TG in liver and their secretion into plasma by the use of palmitic acid- $1-{ }^{14} \mathrm{C}$ and of Triton WR 1339. Several factors have been suggested to be involved in the increased accumulation of triglycerides (TG) in liver observed in several experimental conditions. ${ }^{6)}$ These include (i) enhanced synthesis of TG in liver and their normal or impaired secretion into plasma as lipoproteins (ii) normal synthesis of TG in liver and their impaired secretion into plasma. The secretory rate of hepatic TG into plasma of fed rats with a normal fatty liver has been assessed by the use of labelled palmitate and Triton WR 1339. ${ }^{7)}$ The results of the present investigation show that the release of hepatic TG into plasma of rats fed excess retinol was impaired.

\section{MATERIALS AND METHODS}

Animals and feeding of retinol. Male rats of the
Wistar strain, from the colony maintained in our Institute and weighing $90 \sim 100 \mathrm{~g}$ were used. The animals were divided in two groups each of 12 rats. They were housed in individual cages and were pair fed a stock diet of the following composition: casein $20 \%$, potato starch $70 \%$, Dalda (a hydrogenated vegetable oil) $10 \%$. In addition vitamin mixture, salt mixture and choline chloride at $2.5,5$ and $1.5 \%$ levels respectively were added to the diet. ${ }^{8)}$ The animals had free access to water at all times. To one group of rats $33 \mathrm{mg}$ of retinol (La Roche, Bombay) in groundnut oil was given orally daily for two days. The second group received only the vehicle solution and was treated as the control.

Administration of palmitate- $1-{ }^{14} \mathrm{C}$ and triton WRI339. Twenty four hours after administering the last dose of retinol, rats in both groups were injected plamitic acid$1{ }^{14} \mathrm{C}(10 \mu \mathrm{Ci} / 100 \mathrm{~g}$ body weight, specific activity 5.0 $\mathrm{mCi} / \mathrm{mmole}$ obtained from Bhabha Atomic Research Centre, Bombay) as an albumin complex, ${ }^{9 /}$ through the tail vein under light ether anaesthesia. A $20 \%$ solution of triton WR 1339 (polymeric p-isooctyl polyoxyethylenephenol) (Winthrop Laboratories, New York, U.S.A.) in $0.9 \% \mathrm{NaCl}$ solution $(50 \mathrm{mg} / \mathrm{rat}$ ) was simultaneously injected through the tail vein.

Collection of tissue samples. Three rats in both groups were sacrificed 10,20, 40 and 60 min after the injection of the isotope and of Triton WR 1339. Before sacrifice, blood was collected from the jugular vein into heparinized tubes. The animals were quickly cut open, liver removed, dipped in ice-cold saline, wiped and weighed. Blood was centrifuged for $10 \mathrm{~min}$ at 2000 rpm and plasma decanted off. 
Analytical procedures. Total lipid was extracted from measured aliquots of plasma and liver with chloroform, methanol $(2: 1, v / v){ }^{4}$ Final solution of lipids was made in a known volume of chloroform and a drop of 0.1 percent butylated hydroxy toluene (Nutritional Biochemicals, Cleavland, Ohio, U.S.A.) in chloroform, added and stored at $-20^{\circ} \mathrm{C}$ until used for analyses. Triglycerides, ${ }^{101}$ phosphatidyl choline and phosphatidyl ethanolamine ${ }^{\text {II }}$ were separated by TLC on silica gel $\mathrm{G}$ coated plates. The lipid spots were visualized by a brief exposure to $I_{2}$ vapors. Gel areas corresponding in $R f$ values to standard tripalmitin, phosphatidyl choline (PC) and phosphatidylethanol amine (PE) (Sigma Chemicals, St. Louis, Mo., U.S.A.), cochromatographed with each run, were scraped into tubes. Triglycerides were eluted from the gel with hexane; ether $(1: 1, v / v)$, evaporated to dryness and dissolved in chloroform. One aliquot of the eluates was used for triglyceride estimation ${ }^{121}$ and another aliquot for radioactivity counting. The samples were evaporated to dryness under a stream of air and $15 \mathrm{ml}$ of a scintillation mixture ${ }^{13)}$ was added. Radioactivity was measured in a Packard scintillation Counter. Phospholipids were quantitated by direct digestion of the gel. ${ }^{14}$ For radioactivity determination the phospholipids in the gel were directly transferred into scintillation vials, and $10 \mathrm{ml}$ of scintillation fluid ${ }^{15}$ added. Aliquots of the palmitate-albumin complex were also extracted and counted. Plasma free fatty acids were estimated by the method of Dole and Meinertz, ${ }^{391}$ using palmitic acid as the standard.

The results were calculated and expressed as follows (a) specific activity, counts/min/mg triglycerides, phosphatidyl choline, phosphatidyl ethanolamine, (b) total activities. Liver; $(\mathrm{cpm} / \mathrm{mg}) \times(\mathrm{mg} /$ liver $) \times(100 /$ body-

Table I. Effect of Feeding $33 \mathrm{mg}$ Retinol DaILY For Two Days ON BODY WeIGHT, Liver Weight and Liver Lipids of Rats

Values are mean \pm S.E. from 12 animals in each group. Values significantly different from control are indimated by* $p<0.05$.

\begin{tabular}{lcc}
\hline Body weight $(\mathrm{g})$ & Control & Retinol fed \\
\hline \multicolumn{1}{c}{ Initial } & 94 & 95 \\
$\quad$ Terminal & 91 & 86 \\
Liver weight $(\mathrm{g})$ & 3.28 & 4.26 \\
per $100 \mathrm{~g}$ body weight & 3.81 & $4.95^{*}$ \\
Liver TG & & \\
(mg/100 g body weight) & $9.76 \pm 3.17$ & $21.41 \pm 2.14^{*}$ \\
Liver PC & & \\
(mg/100 g body weight) & $46.65 \pm 3.17$ & $58.65 \pm 2.35^{*}$ \\
Liver PE & & \\
(mg/100 g body weight) & $29.05 \pm 1.9$ & $33.80 \pm 1.90$ \\
Plasma free fatty acids & $749 \pm 42$ & $1405 \pm 38^{*}$ \\
( $\mu$ Eq/lit plasma) & & \\
\hline
\end{tabular}

weight $\times 100 / \mathrm{cpm}$ injected); plasma: $(\mathrm{cpm} / \mathrm{mg}) \times(\mathrm{mg} /$ rat $) \times(100 \times \mathrm{cpm}$ injected $)$, using a plasma volume of $5.46 \mathrm{ml} / 100 \mathrm{~g}$ body weight ${ }^{7)}$ (c) liver: lipids $\mathrm{mg} / 100 \mathrm{~g}$ body weight; plasma: lipids $\mathrm{mg} / 100 \mathrm{ml}$. Results were statistically analysed and differences between the control and retinol fed rats were checked by students ' $t$ ' test and regarded as significant if $P<0.05$.

\section{RESULTS}

The effect of feeding $33 \mathrm{mg}$ of retinol daily for two days on liver TG, PC and PE is shown in Table I. There was a significant increase in liver TG and PC of retinol fed rats as compared to control (Table I). Liver PE contents were not significantly affected in rats fed retinol. Both plasma TG and PC were significantly high in rats fed retinol as compared to control (Fig. 1).

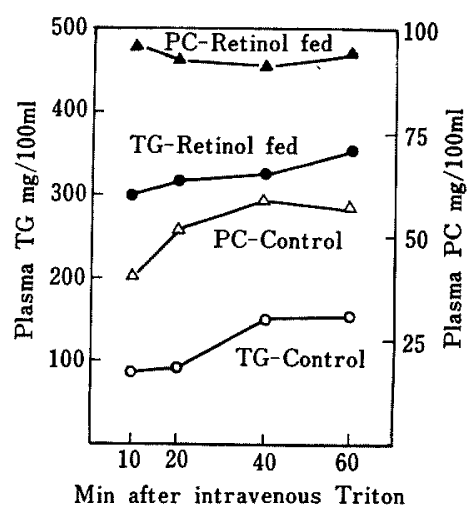

FIG. 1. Effect of Feeding $33 \mathrm{mg}$ of Retinol Daily for Two Days on Plasma Triglycerides and Phosphatidyl Choline Content of Rats.

Values are mean of 12 animals in each group.

Incorporation of palmitic acid- $1-{ }^{14} \mathrm{C}$ into liver and plasma $T G$

Incorporation of palmitic acid- $1-{ }^{14} \mathrm{C}$ into liver total lipids of control and retinol fed rats at 10,20,40 and $60 \mathrm{~min}$ after the injection of the isotope and of Triton is shown in Fig. 2. The incorporation of palmitic acid- $1-{ }^{14} \mathrm{C}$ into total lipids was significantly higher in retinol fed rats at all periods of study as compared to control. The maximum incorporation of the isotope into liver lipids was at $10 \mathrm{~min}$ period. The rise and decline of the radioactivity of 
liver lipids of control was very rapid. However in the retinol fed rats the decline of the radioactivity was slower (Fig. 2). Incorporation of

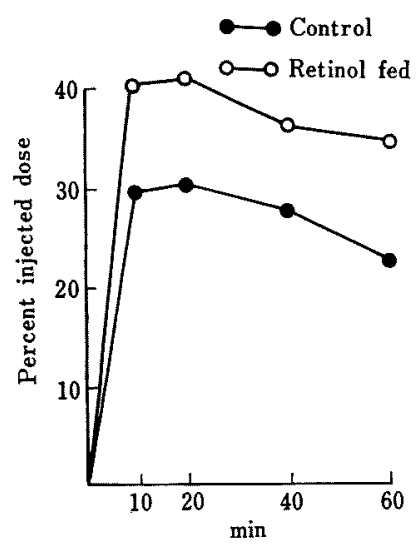

Fig. 2. Palmitic Acid-1-14 C Incorporation into Liver Lipids (total activity $=(\mathrm{cpm} / \mathrm{mg}$ lipid $) \times(\mathrm{mg} /$ liver $) \times$ $100 /$ body weight $\times 100 / \mathrm{cpm}$ injected).

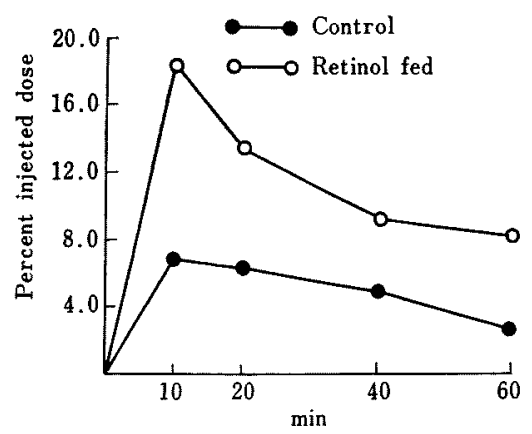

FIG. 3. Plamitic Acid-1-14 C Incorporation into Liver Triglycerides (total activity $=(\mathrm{cpm} / \mathrm{mg}$ triglycerides $) \times$ $(\mathrm{mg} /$ liver $) \times 100 /$ body weight $100 / \mathrm{cpm}$ injected $)$.

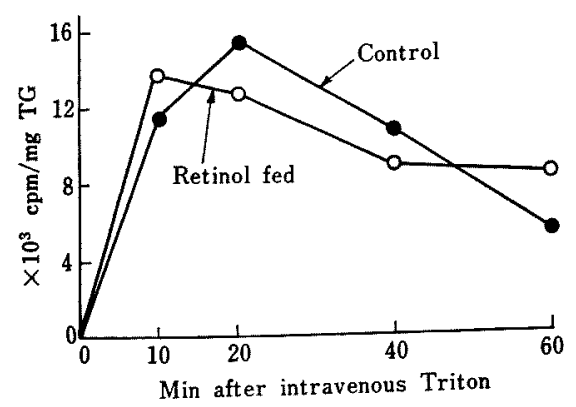

FIG. 4. Palmitic Acid-1-14C Incorporation into Liver Triglycerides (specific activity $=$ counts $/ \mathrm{min} / \mathrm{mg}$ triglycerides of rats orally fed $33 \mathrm{mg}$ of retinol daily for two days. Values are mean of 3 animals in each group. palmitic acid- $1-{ }^{14} \mathrm{C}$ into liver TG of control and retinol fed rats is shown in Figs. 3 and 4. Total activity (percent injected dose) of liver TG of retinol fed rats, which was maximum at $10 \mathrm{~min}$ period was significantly higher than that of control (Fig. 3). However the specific activities (counts $/ \mathrm{min} / \mathrm{mg}$ ) of liver TG in retinol fed and control rats were not significantly different (Fig. 4) suggesting that the higher total activity of liver TG observed in retinol fed rats (Fig. 3) might have resulted from retention of the newly incorporated label into TG of these rats.

The palmitic acid- $1-{ }^{14} \mathrm{C}$ radioactivity (percent injected dose) and specific activity (counts/ $\mathrm{min} / \mathrm{mg}$ ) appearing in plasma TG of both reti-

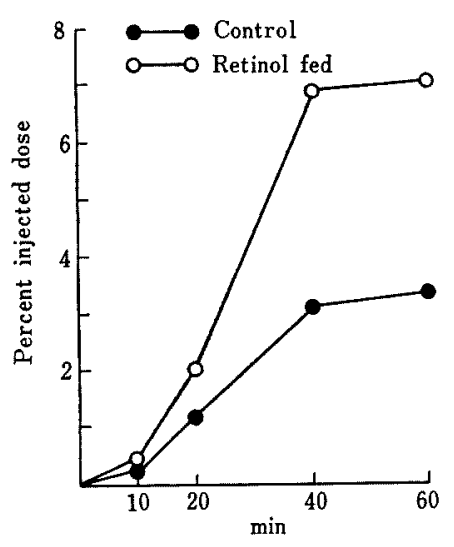

FIG. 5. Incorporation of Palmitic Acid-1 ${ }^{-14} \mathrm{C}$ into Plasma Triglycerides (total activity $=(\mathrm{cpm} / \mathrm{mg}$ triglycerides $) \times(\mathrm{mg} / \mathrm{rat}) \times 100 / \mathrm{cpm}$ injected. $)$

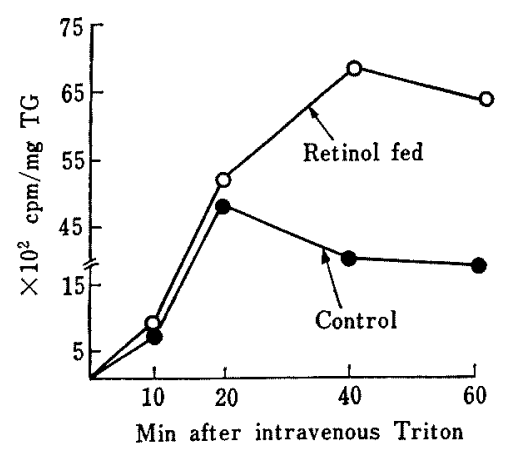

FIG. 6. Palmitic Acid-1-14C Incorporation into Plasma Triglycerides (specific activity $=$ counts $/ \mathrm{min} / \mathrm{mg}$ triglycerides). 
nol fed and control rats is shown in Figs. 5 and 6 . The accumulation of radioactivity into plasma $T G$ which reached maximum at $40 \mathrm{~min}$ was significantly higher in retinol fed rats as compared to control. In tritonized control rats (Fig. 1) plasma TG contents were almost doubled at $60 \mathrm{~min}$ after its administration whereas in retinol fed rats, no such increase was observed, but the plasma TG values in retinol fed rats remained higher than control at all periods (Fig. 1).

Incorporation of palmitic acid- $1-{ }^{14} \mathrm{C}$ into liver and plasma $P C$ and $P E$

Labelling pattern with time of liver $\mathrm{PC}$ and $\mathrm{PE}$ of control and retinol fed rats is shown in Figs. $7 \sim 10$. Maximum incorporation of the radioactivity occurred at $10 \sim 20 \mathrm{~min}$ in both

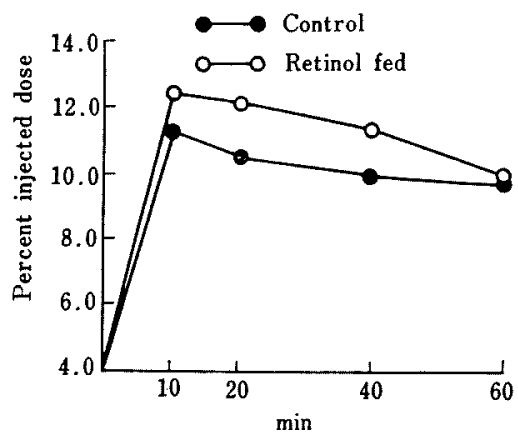

FIG. 7. Palmitic Acid-1-14C Incorporation into Liver Phosphatidyl Choline (total activity $=(\mathrm{cpm} / \mathrm{mg} \mathrm{mg} \mathrm{Pc})$ $\times(\mathrm{mg} /$ liver $) \times(100 /$ body weight $\times 100 / \mathrm{cpm}$ injected $))$.

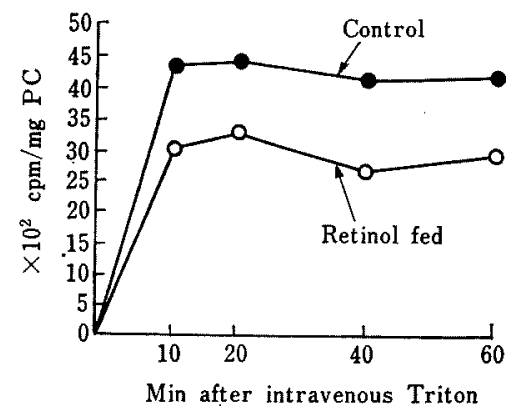

FIG. 8. Palmitic Acid-1-14 C Incorporation into Liver Phosphatidyl Choline (specific activity $=\mathrm{cpm} / \mathrm{mg}$ phosphatidyl choline) of rats orally fed $33 \mathrm{mg}$ of retinol daily for two days. Values are mean of 3 animals in each group.
$\mathrm{PC}$ and PE fraction but the accumulation of radioactivity (percent injected dose) in $\mathrm{PC}$ and $\mathrm{PE}$ of retinol fed rats was more as compared to control (Figs. 7 and 9). The loss of radio-

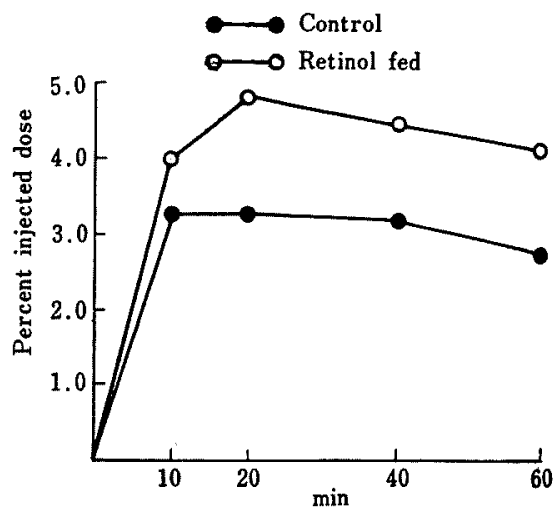

FIG. 9. Incorporation of Palmitic Acid-1-14C into Liver Phosphatidyl Ethanolamine (total activity= $(\mathrm{cpm} / \mathrm{mg} \mathrm{PE}) \times(\mathrm{mg} /$ liver $) \times 100 /$ body weight $\times 100 /$ cpm injected).

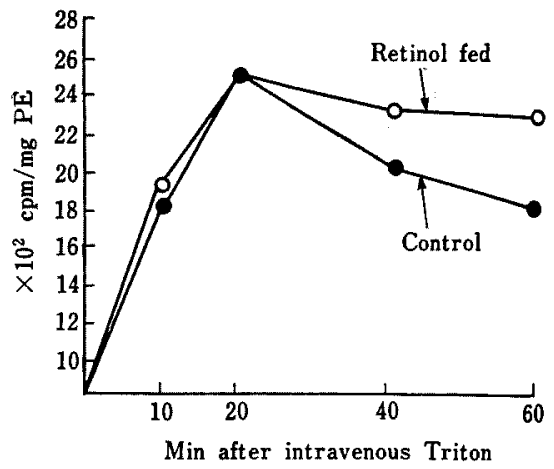

Frg. 10. Palmitic Acid-1-14C Incorporation into Liver Phosphatidyl Ethanolamine (Specific activity= counts $/ \mathrm{min} / \mathrm{mg}$ phosphatidyl ethanolamine).

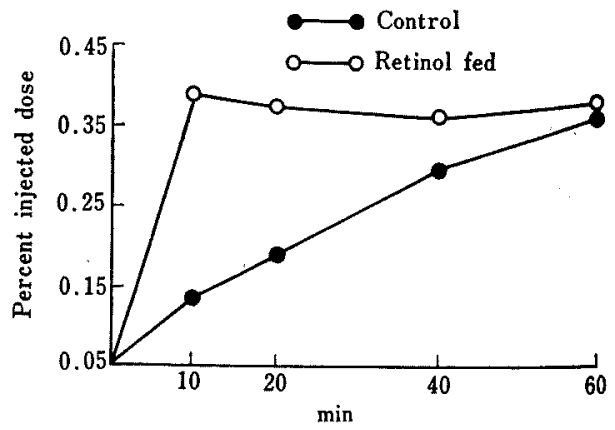

FIG. 11. Palmitic Acid-1-14 C Incorporation into Plasma Phosphatidyl Choline (total activity $=(\mathrm{cpm} / \mathrm{mgPc}$ ) $\times(\mathrm{mg} / \mathrm{rat}) \times 100 / \mathrm{cpm}$ injected $)$. 


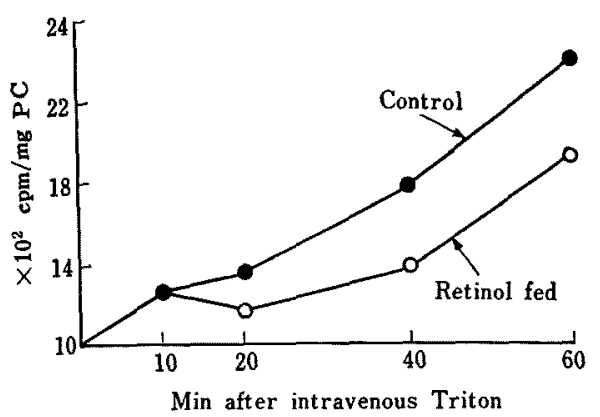

Fig. 12. Palmitic Acid-1 - ${ }^{14} \mathrm{C}$ Incorporation into Plasma Phosphatidyl Choline (specific activity $=$ counts/ $\mathrm{min} / \mathrm{mg}$ phosphatidyl choline) of rats orally fed $33 \mathrm{mg}$ of retinol for two days. Values are mean of 3 animals in each group.

activity was faster in PC than in PE of retinol fed rats as compared to control, the amount of palmitic acid-1- ${ }^{14} \mathrm{C}$ labelled PC (percent injected dose) appearing in plasma was signifcantly higher in retinol fed rats as compared to control (Fig. 11). In retinol fed rats the maximum rise in $\mathrm{PC}$ radioactivity was at $20 \mathrm{~min}$ and after that it plateaued off whereas in control rats this rise was slow and gradual, reaching the values of retinol fed rats at $60 \mathrm{~min}$ after the injection of the isotope (Fig. 12).

\section{DISCUSSION}

Administration of retinol either orally or intramuscularly for varying periods to rats fed ad libitum has been reported to increase liver triglycerides and cause hypertriglyceridemia and hyperphospholipedemia. ${ }^{3 \sim 5)}$ Increase in plasma triglycerides and phospholipids has also been reported in humans given large quantity of retinol. ${ }^{16,17)}$ The results of the present investigation confirm that oral administration of $33 \mathrm{mg}$ retinol daily for two days causes an accumulation of triglycerides and phosphatidyl choline in liver and plasma as compared to the controls (Table I and Fig. 1). Several conditions of triglyceride metabolism in liver can lead to their accumulation under a variety of experimental manipulations. ${ }^{6)}$ Some of these include (a) an enhanced synthesis of triglyceride and their normal or impaired secretion to plasma and (b) normal synthesis of trigly- cerides and their impaired secretion into plasma. In the reported experiments the above two aspects of triglyceride metabolism have been studied in rats fed retinol. The incorporation of palmitate- $1-{ }^{14} \mathrm{C}$ into liver total lipids triglycerides (Fig. 2 and Fig. 3) of retinol fed rats was significantly higher at all periods studied, after the injection of the isotope as compared to control. However the specific activities (counts/min/mg TG) of TG in control and retinol fed rats were nearly comparable at all periods of study, suggesting that the rate of the synthesis of TG in the two groups was not different (Fig. 4). However if we consider the enlarged pool size of liver TG in retinol fed rats (Table I), then it is noted that inspite of the dilution of the label by the TG pool of retinol fed rats, the specific activity (counts/ $\mathrm{min} / \mathrm{mg} \mathrm{TG}$ ) of $\mathrm{TG}$ was comparable to the controls, showing that the rate of synthesis of TG in retined fed rats was greater than the controls (Fig. 4). The loss of label from liver TG would indicate its utilization. The results presented in Fig. 3 point out that though the utilization of newly synthesized TG in control and retinol fed rats was nearly comparable, still the $T G$ radioactivity retained in liver of retinol fed rats was higher than the controls. The results suggest that a increased synthesis and an impaired secretion of liver $T G$ in retinol fed rats lead to their increased accumulation as compared to the controls. An impairment in the release of hepatic $T G$ is usually reflected by lower concentration of plasma TG. ${ }^{18}$ To assess the ability of liver to secrete TG into plasma in a variety of experimental conditions use of Triton WR 1339 , a nonionic detergent has been made. ${ }^{19 \sim 22)}$ To circumvent the many limitations of the use of this test in fed rats, Lombardi et al. performed this test to estimate liver TG secretion in choline deficient rats by simultaneously injecting labelled palmitic acid and Triton-WR $1339 .{ }^{7}$ To assess the ability of liver to secrete TG into plasma of retinol fed rats, both palmitic acid- $1-{ }^{14} \mathrm{C}$ and Triton-WR 1339 were simultaneously injected and increment in the radioactivity of plasma $\mathrm{TG}$ with time measured. 
The results showed that at all periods the palmitate- $1-{ }^{14} \mathrm{C}$ radioactivity of plasma $\mathrm{TG}$ of retinol fed rats was consistently higher than the control (Fig. 5). The specific activities (counts $/ \mathrm{min} / \mathrm{mg}$ ) of plasma TG of control and retinol fed rats (Fig. 6) show that the turnover rate of plasma $\mathrm{TG}$ in retinol fed rats was slower than that of the controls. These results point out an impairment in the uptake of plasma TG in retinol fed rats by extrahepatic tissues. Intravenous administration of Triton into animals causes marked hyperlipemia and hypertriglyceredemia. ${ }^{23 \sim 24)}$ This effect results from a block in the exit of lipids from plasma compartment due to alterations in the physiochemical properties of plasma lipoproteins. ${ }^{25}$ ) From the results shown in Fig. 1, it is seen that in control animals $60 \mathrm{~min}$ after the administration of Triton, plasma TG increased by about two fold whereas no such increase was noted in retinol fed rats. Possible explanation for this could be that retinol fed rats were already hypertriglyceredemic and thus only a slight increase in their plasma TG after the administration of Triton could be observed. The hyperlipemia and hypertriglyceredemia observed in retinol fed rats could also result from their decreased utilization by extrahepatic tissues.

In experimental conditions where an increased accumulation of TG in liver has been reported, an altered metabolism of liver phospholipids has also been observed. ${ }^{7}$ In retinol fed rats liver contents of phosphatidyl choline were significantly higher and that of phosphatidyl ethanolamine unchanged as compared to control (Table I). Results of incorporation of palmitic acid- $1-{ }^{14} \mathrm{C}$ into phosphatidyl choline (percent injected dose) show that extent of the label in PC of retinol fed rats were significantly higher than the controls (Fig. 7). However the specific activity of liver PC (counts/min/ $\mathrm{mg}$ ) of retinol fed rats was considerably lower than the controls (Fig. 8) indicating that its synthesis from palmitate- $1-{ }^{14} \mathrm{C}$ was reduced in retinol fed rats and the increased radioactivity observed in PC (percent injected dose) in retinol fed rats (Fig. 7) results from their impaired secretion into plasma. Both the total palmitate- $1-{ }^{14} \mathrm{C}$ radioactivity (percent injected dose, Fig. 9) and the specific activity (counts/ $\mathrm{min} / \mathrm{mg}$ ) of liver PE (Fig. 10) in retinol fed rats were higher than the control. These results suggest that the total activity of PE may result from its impaired secretion as well as slower turnover rate in liver of retinol fed rats as compared to the controls. Both PC and PE are the major phospholipids of liver constituting about $70 \sim 80$ percent of liver total phospholipids. In plasma, PC forms about 60 percent of plasma phospholipids which are derived from liver, but PE forms only a small fraction of plasma phospholipids. Therefore, the measurement of the secretion of plasma PC in Tritonized rats could also provide an indirect information on hepatic TG secretion. ${ }^{3)}$ The results showed that in control rats $60 \mathrm{~min}$ after the administration of Triton there was a 38 percent increase in plasma $\mathrm{PC}$, whereas no such increase was noted in retinol fed rats (Fig. 1). Like plasma TG, the values of plasma $P C$ were about 2 fold higher in retinol fed and tritonized rats as compared to control (Fig. 1). Therefore, due to already expanded pool of plasma PC in retinol fed rats any increase in plasma $P C$ due to Triton administration may not be evident. These results suggest an impaired secretion of liver TG and PC into plasma of retinol fed rats. The increased levels of TG and PC may have resulted from their impaired uptake by extrahepatic tissues. The total activity (percent injected dose) of palmitate- $1{ }^{14} \mathrm{C}$ appearing in plasma $\mathrm{PC}$ of retinol fed rats was higher than the control (Fig. 11), but the specific activity was lower than the control (Fig. 12). The former results suggest a slower loss of the PC label in plasma of retinol fed rats (Fig. 11), perhaps by its impaired uptake by extrahepatic tissues, resulting in the accumulation of newly secreted labelled PC in plasma of retinol fed rats. The lowered specific activity of plasma $\mathrm{PC}$ in retinol fed rats results from its expanded pool size. It may be noted that there are differences between increase in plasma TG and PC content in control tritonized rats. There was about 
two fold increase in TG, whereas in PC only 38 percent increase was noted (Fig. 1). The reasons for this are a considerably slower turnover of plasma PC and the fact that among the plasma lipoproteins the very low density lipoproteins fraction has the fastest turnover but the lowest content of phospholipids.

Results of this study show that the elevated levels of triglycerides in liver of retinol fed rats as compared to control result from their increased synthesis and impaired secretion into plasma. Hepatic triglycerides are synthesized from fatty acids and $\alpha$-glycerophosphate. ${ }^{27}$ ) The rate of synthesis is directly proportional to the concentration of both the substrates. ${ }^{28 \sim 30}$ ) The significantly elevated levels of plasma-non esterified fatty acids in retinol fed rats as compared to control (Table I) show that liver utilizes fatty acids for TG synthesis which are mobilized from adipose tissues. Hence in conditions of extensive lipolysis it is reasonable to assume that the flux of free fatty acid to liver is enhanced. ${ }^{31} \quad$ Evidence to this assumption is provided by the specific activities (counts $/ \mathrm{min} / \mathrm{mg}$ ) data of liver TG in control and retinol fed rats (Fig. 4), which were not different, inspite of the fact that the palmitate$1{ }^{-14} \mathrm{C}$ radioactivity of plasma free fatty acids (precursor pool of liver fatty acids) in retinol fed rats was considerably diluted due to its expanded pool size as compared to the control (Table I). Under these conditions, to achieve the synthetic rate of liver TG in retinol fed rats similar to that of control, the flux of plasma free fatty acids to liver in retinol fed rats has to be more. The amounts of $\alpha$-glycerophosphate needed for TG synthesis may be provided by glycolytic and/or gluconeogenic pathway or by the glycerokinase reactions. ${ }^{32 \sim 33)}$ Corticosteroids have been implicated to be involved in the liver triglyceride metabolism. Corticosteroid administration caused increased synthesis of triglycerides in liver and produced hypertriglyceridemia. $^{34,35)}$ Studying protein synthesis in liver ${ }^{36)}$ and cholesterogenesis in adrenals of rats given vitamin $\mathrm{A}$ and vitamin $\mathrm{C},{ }^{37,38)}$ we have suggested that the effect of vitamin A on liver lipid metabolism are mediated through the adrenals. The results of the present investigation further suggest that the effects of vitamin $\mathrm{A}$ on liver triglycerides are mediated through the adrenals.

Acknowledgements. This work was supported in part by the PL-480 funds of the U.S. Department of Agriculture, Washington D.C. (grant No. Project No. A7-HN-22). Retinol used was kindly supplied by $\mathrm{M} / \mathrm{s}$. La Roche, Bombay.

\section{REFERENCES}

1) T. Moore, "Vitamin A," Elsevier Publishing Co., Amsterdam, 1957, p. 340.

2) A. Ray and D. P. Sadhu, Nature, 184, 1323, (1959).

3) U. K. Misra, Nature, 209, 910 (1966).

4) U. K. Misra, Canad. J. Biochem., 46, 697 (1968).

5) U. K. Misra, Agr. Biol. Chem., 32, 707 (1968).

6) B. Lombardi, Lab. Invest., 15, 1 (1966).

7) B. Lombardi, P. Pani and F. F. Schlunk, J. Lipid Res., 9, 437 (1968).

8) A. E. Harper, $J$. Nutri., 68, 405 (1959).

9) I. B. Fritz, Am. J. Physiol., 197, 297 (1959).

10) U. K. Misra, Biochem. Biol. Speri. VII, 1, 2, 57 (1968).

11) U. K. Misra, Biochem. Biol. Speri. VT, 4, 199 (1957).

12) E. VanHandel and D. E. Zilversmit, J. Lab. Clin Invest., 50, 152 (1957).

13) F. Snyder and M. Stephens, Anal. Biochem., 4, 128 (1962).

14) U. K. Misra, Biochim. Biol. Speri. VIII, No. 2, 125 (1969).

15) F. Snyder, Anal. Biochem., 9, 183 (1964).

16) J. T. Van Bruggen and J. V. Strumfjord, J. Lab. Clin. Med., 33, 67 (1948).

17) M. Pallotatta and R.F. Krause, Am. J. Clin. Nutri., 13, 201 (1963).

18) R. C. Recknagel, B. Lombardi and M. C. Schotz, Proc. Soc. Exptl. Biol. Med., 104, 608 (1960).

19) M. Friedman, and S. O. Byers, Am. J. Physiol., 190, 439 (1957).

20) S. Otway and D. S. Robinson, J. Physiol. (London) 190, 321 (1967).

21) R. O. Recknagel Pharmacol. Rev., 19, 145 (1967).

22) B. Lombardi and R. O. Recknagel, Am. J. Pathol, 40, 571 (1962).

23) A. Kellner, J. W. Correll and A. T. Ladd, J. Exp. Med., 93, 373 (1951).

24) M. Friedman, and S. O. Byers, ibid., 97, 117 (1953).

25) A. Scanu and P. Oriente, ibid., 113, 735 (1961).

26) M. C. Fishler, C. Entenman, M. L. Montgomery and I. L. Chaikoff, J. Biol. Chem., 150, 47 (1943). 
27) E. P. Kennedy, Fed. Proc. Fed. Amer. Soc. Exp. Biol., 20, 934 (1961).

28) R. Tzur, E. Tal and B. Shapiro, Biochim. Biophys. Acta, 84, 18 (1964).

29) E. A. Nikkila and K. Ojala, Life Sci., 3, 1021 (1964).

30) E. A. Nikkila and K. Ojala, ibid., 4, 937 (1965).

31) B. B. Brodie, J. I. Davies, S. Hynie, G. Krishna and B. Weiss, Pharmac. Rev., 18, Part I, 273 (1966)

32) Y. Stein and B. Shapiro, Biochim, Biophys. Acta, 24, 197 (1957).

33) C. Bublitz and E. P. Kennedy, J. Biol. Chem., 211, 951 (1954).
34) R. P. Maickel and B. B. Brodie, Ann. N. Y. Acad. Soc., 104, 1059 (1963).

35) E.P. Reaven, O.G. Kolterman and G.M. Reaven, J. Lipid Res., 15, 74 (1974).

36) H. C. Ahuja and U. K. Misra, Nut. Rept. Intern., 7, 85 (1973).

37) A. K. Mathur, R. Ramanathan and U. K. Misra, Int. J. Vit. Nut. Res., 44, 19 (1974).

38) U. K. Misra, and N. Srivastava, ibid., 44, 175 (1974).

39) V. P. Dole and H. Meinertz, J. Biol. Chem., 233, 2595 (1960). 